nicht aus dem gewanschten Salze, sondern wesentlich aus neutralem Magnesiumsulfit.

\section{Versuche zur Darstellung fester Zink-} salze der unterschwefligen Saure.

Der Elektrolyt wurde gewonnen durch Einleiten von $\mathrm{SO}_{2}$ in einer Aufschlammung von ro $\mathrm{g} \mathrm{ZnCO} \mathrm{CO}_{3}$ in $100 \mathrm{~cm} \mathrm{H}_{2} \mathrm{O}$. Bei der Elektrolyse mit und ohne Diaphragma, mit und ohne Einleitung von $\mathrm{SO}_{2}$, bei Verwendung von Elektroden aus $P t$ wie aus $Z n$ gelang es nicht, eine Ausscheidung von festem Zinkhydrosulfit zu erzielen. Wenn ein Niederschlag ausfiel, bestand er aus neutralem Zinksulfit, in manchen Fallen verunreinigt durch kathodisch abgeschiedenes Zink, ausnahmsweise auch durch Schwefel.

Da sich häufig Doppelsalze leichter abscheiden als die entsprechenden einfachen Salze, wurden Lossungen elektrolysiert, welche die sauren Sulfite von Natrium und Zink enthielten. Hierbei fielen Niederschläge aus, welche neben schwefligsaurem Zink und metallischem Zink reichlich Hydrosulfit, und zwar hauptsåchlich als Natriumsalz enthielten. Bei der Elektrolyse einer Lósung, die in $100 \mathrm{ccm} 33,5 \mathrm{~g} \mathrm{NaHSO}$ und $65,0 \mathrm{~g}$ $\mathrm{Zn}\left(\mathrm{HSO}_{3}\right)_{2}$ enthielt, unter Anwendung eines Diaphragmas und einer Kathode aus amalgamiertem Zinkblech, hatte sich eine breiige, schmutzigweisse Masse schon nach Zufuhr von I Amp.-Stunde so dicht abgeschieden, dass der Stromdurchgang erschwert und deshalb der Versuch abgebrochen wurde. Der Niederschlag wog nach dem Filtrieren und Trocknen unter Luftabschluss $12 \mathrm{~g}$ und bestand zu reichlich ein Viertel seines Gewichtes aus Natriumhydrosulfit, was einer Stromausbeute von rund $86 \%$ entspricht; bedenkt man, dass auch noch etwas Hydrosulfit in der Losung bleibt, so stimmt diese Ausbeute aberein mit derjenigen, welche man an gelöstem Hydrosulfit bei der Elektrolyse von Natriumbisulfit anfanglich erhält. Erniedrigt man den Gehalt der Kathodenflussigkeit an Zinkbisulfit und erboht den an Natriumbisulfit, so kann man länger Strom zufahren, ehe durch Abscheidung fester Salze der Widerstand des Bades zu sehr steigt; die gewonnene Substanzmenge wächst dadurch, die Stromausbeute aber nimmt $a b$.

Versuche mit Losungen von Natrium-Magnesiumbisulfit und Natrium-Manganbisulfit ergaben wohl gelostes Hydrosulfit, aber keinerlei Abscheidungen von Salzen.

\section{Zusammenfassung.}

I. Zur Darstellung konzentrierter Lösungen von Hydrosulfiten ist die rein chemische Reduktion schwefligsaurer Salze geeigneter als die elektrolytische.

2. Die Gewinnung fester Hydrosulfite gelingt durch kathodische Reduktion einer NatriumZink-Bisulfitlosung; dieser Weg ist aber viel ungunstiger als der rein chemische.

3. Die Angaben des D. R.-P. Nr. 125207 von A. R. Frank sind in allen wesentlichen Punkten unrichtig; man kann nach den gegebenen Vorschriften weder das Calciumsalz, noch das Magnesiumsalz der unterschwefligen Saure in festem Zustande herstellen.

4. Verdünnte Lơsungen von Natriumhydrosulfit lassen sich durch elektrolytische Reduktion saurer schwefligsaurer Salze mit vorzäglicher Ausbeute gewinnen; wenn diese Losungen von geringem Gehalte sofort zu Reduktionswirkungen verwendet werden, also beispielsweise nur als Ueberträger der kathodischen Reduktionswirkung dienen, so kann die elektrochemische Erzeugung von Hydrosulfiten vorteilhaft sein. Dieser Fall liegt vor bei dem D. R. - P. Nr. 139567 (1902) der Farbwerke vorm. Meister Lucius \& Braning, Verfahren zur Reduktion von Indigo. Hier wird durch kathodische Reduktion von Bisulfit Hydrosulfit erzeugt, welches sofort zur Bildung von Indigoweiss verbraucht und von der Kathode stetig nachgeliefert wird.

Giessen, im April 1904.

Physikalisch - Chemisches Univers. -Laboratorium. (Eingegangen: 22. Aprii.)

\title{
ANODISCHES VERHALTEN VON ZINN, ANTIMON UND WISMUT.
} Von $K$. Elbs und H. Thümmel.

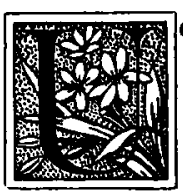

eber die anodische Auflösung von Metallen mit wechselnder Wertigkeit liegen schon viele Untersuchungen vor ${ }^{1}$ ); unsere Mitteilung bildet einen weiteren Beitrag zur Kenntnis dieser Vorgänge und erstreckt sich auf Anoden aus

I) W. Bolton, Zeitschr. f. Elektrochemie 2, 73 bis 79; 93 bis 98 ; 183 bis $190(1895)$. R. Lorenz, Zeitschr. f. anorgan. Chemie 12, 436 (1896). W. Borchers, Zeitschr.f. Elektrochemie 3, 482 bis 485 (1896). K. E1bs
Zinn, Antimon und Wismut in wässerigen Lossungen von Chlornatrium, Natriumsulfat, Chlorwasserstoffsäure und $\mathrm{Sch}$ wefelsäure als Elektrolyten.

Die Anoden wurden in Stabform verwendet; ihre wirksame Oberflache liess sich durch einen

und F. Fischer, Zeitschr. f. Elektrochemie 7, 343 bis 347 (1900). K. Elbs, Zeitschr. f. Elektrochemie 8, 512 (I902). K. Elbs und J. Forssell, Zeitschr. f. Elektrochemie 8, 760 bis 772 (I902). 
abergezogenen Gummischlauch regeln. Als Kathode diente stets Platin, als Bad ein hohes Becherglas, von dessen Boden die Elektroden einige Centimeter Abstand hatten, so dass die Niederschläge sich ungestört absetzen konnten. Als Material kam in Verwendung:

Zinn, gereinigt, in Stangen, von der chemischen Fabrik C. A. F. Kahlbaum, Berlin.

Antimon, chemisch rein, in Stangen, von der chemischen Fabrik E. de Haën, Hannover.

Wismut, chemisch rein, von der chemischen Fabrik G. Throm, Giessen.

Das Kahlbaumsche gereinigte Zinn erwies sich als so rein, dass es die Marke „Chemisch reines Zinn" völlig ersetzen konnte:

0,42 I 9 getall hinterliessen beim Lösen in Salzsäure einen sehr geringen kohligen Räckstand und lieferten:

0,5340 g $\mathrm{Sn}_{n} \mathrm{O}_{2}$, entsprechend $99,6 \% \mathrm{Sn}$, o,0007 " $\mathrm{Fe}_{2} \mathrm{O}_{3}, \quad$ o, I \% $\mathrm{Fe}$, kohligen Rückstand, " $0,3 \%$.

Die beiden anderen Metalle entsprachen der Bezeichnung "chemisch rein".

\section{Anodisches Verhalten des Zinns.}

Bei der leichten Oxydierbarkeit der meisten Verbindungen des zwciwcrtigen Zinns bewährte sich folgender Weg der Analyse am besten: Elektrolyt samt darin aufgeschlämmtem Niederschlag wurde nach beendigter Elektrolyse mit einem Ueberschuss von verdünnter Schwefelsäure versetzt, die klare Lösung heiss mit Kaliumpermanganat titriert, woraus sich der Gehalt an zweiwertigem Zinn ergibt, dann in der titrierten Lösung nach der Methode von A. Classen (Zusatz von Ammoniumoxalat und Oxalsäure) der Gesamtzinngehalt bestimmt durch Niederschlagung auf eine Messingdrahtnetzelektrode.

Für den raschen Verlauf der Titration war der geringe Gehalt von o, I \% Eisen wesentlich, da andernfalls gegen Ende die Oxydation zu träge wird. War Chlornatrium oder Salzsäure als Elektrolyt verwendet, so wurde vor der Titration ein Zusatz von Mangansulfat gemacht, um wenigstens eine annähernd richtige $\mathrm{Be}$ stimmung zu gewährleisten.

Je nach Umständen betrug die Stromzufuhr o,4 bis 3,0 Amp.-Stunden; Anode und Kathode waren mit dickem, lockerem Baumwollgewebe umhallt, um ein Abfallen von losen Teilchen zu verhindern.

Bei Chlornatrium und Natriumsulfat als Elektrolyten blieben die Lösungen neutral, ein Beweis, dass die Niederschläge keine basischen Salze, sondern reine Hydroxyde enthielten. Natriumnitrat empfiehlt sich nicht als Elektrolyt, da es in der Kälte mässig, in der Hitze stark der kathodischen Reduktion unterliegt.
Tabelle I.

\begin{tabular}{|c|c|c|c|c|c|}
\hline \multirow{2}{*}{ Elektrolyt } & \multirow{2}{*}{$\begin{array}{l}\text { Temperatur } \\
\text { in } \\
\text { Grad C. }\end{array}$} & \multirow{2}{*}{$\begin{array}{c}\text { Strom- } \\
\text { dichte } \\
\text { in } \\
\text { Amp/qcm }\end{array}$} & \multicolumn{2}{|c|}{$\begin{array}{l}\text { Anodisch } \\
\text { gelostes } S n \\
\text { in Gramm }\end{array}$} & \multirow{2}{*}{$\begin{array}{c}\text { Zwei- } \\
\text { wertiges } \\
\text { Sn in } \\
\text { Prozent } \\
\text { der } \\
\text { Gesamt- } \\
\text { menge } \\
\end{array}$} \\
\hline & & & $\begin{array}{c}\text { Gesamt- } \\
\text { menge }\end{array}$ & $\mid \begin{array}{c}\text { zwei- } \\
\text { wertiges } \\
5 n\end{array}$ & \\
\hline $\begin{array}{l}\text { 2 n. } N a C l- \\
\text { Lösung, d. h. } \\
\text { in I Liter } \\
\text { I I } 7 \mathrm{~g} \mathrm{NaCl}\end{array}$ & $\begin{array}{c}18 \\
18 \\
50 \\
95-100 \\
95-100 \\
95-100\end{array}$ & $\begin{array}{r}I \\
10 \\
100 \\
1 \\
10 \\
100\end{array}$ & $\begin{array}{l}0,0943 \\
0,0930 \\
0,0653 \\
0,1142 \\
0,1190 \\
0,1082\end{array}$ & $\begin{array}{l}0,0932 \\
.0,0929 \\
0,0919 \\
0,1056 \\
0,1150 \\
0,1013\end{array}$ & $\begin{array}{l}98,8 \\
99,8 \\
96,4 \\
92,4 \\
96,6 \\
93,6\end{array}$ \\
\hline $\begin{array}{l}\text { O, I n. } N a C l- \\
\text { Lösung, d. h. } \\
\text { in I Liter } \\
5,85 \mathrm{~g} \mathrm{NaCl}\end{array}$ & $\begin{array}{c}18 \\
18 \\
95-100 \\
95-100 \\
\end{array}$ & $\begin{array}{r}\text { I } \\
\text { IO } \\
\text { I } \\
\text { IO }\end{array}$ & $\begin{array}{l}0,1020 \\
0,1007 \\
0,0959 \\
0,1021\end{array}$ & $\mid \begin{array}{l}0,0927 \\
0,0972 \\
0,0942 \\
0,0989\end{array}$ & $\begin{array}{l}90,8 \\
96,5 \\
98,2 \\
96,8\end{array}$ \\
\hline $\begin{array}{l}2 \mathrm{n.} N a_{2} \mathrm{SO}_{4} \\
\text { Iösung, d. h. } \\
\text { in I Liter } \\
\text { I } 42 \mathrm{~g} N a_{2} \mathrm{SO}_{4}\end{array}$ & $\begin{array}{c}18 \\
18 \\
50 \\
95-100 \\
95-100 \\
95-100\end{array}$ & $\begin{array}{r}1 \\
10 \\
100 \\
1 \\
10 \\
100\end{array}$ & $\begin{array}{l}0,0934 \\
0,0973 \\
0,1130 \\
0,1042 \\
0,1189 \\
0,1182\end{array}$ & $\begin{array}{l}0,0869 \\
0,0917 \\
0,1056 \\
0,0992 \\
0,1181 \\
0,1125\end{array}$ & $\begin{array}{l}93,0 \\
94,2 \\
93,4 \\
95,2 \\
99,3 \\
95,1\end{array}$ \\
\hline $\begin{array}{l}\text { o, I n. } N a_{2} S O_{4^{-}} \\
\text {Lösung, d. h. } \\
\text { in I Liter } \\
\text { 7, I g } N a_{2} S O_{4}\end{array}$ & $\begin{array}{c}18 \\
18 \\
95-100 \\
95-100\end{array}$ & $\begin{array}{r}1 \\
\text { ro } \\
1 \\
10\end{array}$ & $\begin{array}{l}0,0986 \\
0,0947 \\
0,0482 \\
0,1081\end{array}$ & $\left|\begin{array}{l}0,0942 \\
0,0942 \\
0,0457 \\
0,1079\end{array}\right|$ & $\begin{array}{l}95,5 \\
99,4 \\
94,8 \\
99,8\end{array}$ \\
\hline
\end{tabular}

Tabelle 2.

\begin{tabular}{|c|c|c|c|c|c|}
\hline \multirow{2}{*}{ Elektrolyt } & \multirow{2}{*}{$\begin{array}{c}\text { Temperatur } \\
\text { in } \\
\text { Grad } \mathrm{C} .\end{array}$} & \multirow{2}{*}{$\begin{array}{c}\text { Strom- } \\
\text { dichte } \\
\text { in } \\
\text { Amp/qcm }\end{array}$} & \multicolumn{2}{|c|}{$\begin{array}{l}\text { Anodisch } \\
\text { gelostes } S_{n} \\
\text { in Gramm }\end{array}$} & \multirow{2}{*}{$\begin{array}{c}\text { Zwei- } \\
\text { wertiges } \\
\text { Sn in } \\
\text { Prozent } \\
\text { der } \\
\text { Gesamt- } \\
\text { menge }\end{array}$} \\
\hline & & & $\begin{array}{c}\text { Gesamt- } \\
\text { menge }\end{array}$ & $\left|\begin{array}{c}\text { zwei- } \\
\text { wertiges } \\
S n\end{array}\right|$ & \\
\hline $\begin{array}{l}2 \mathrm{n.} \mathrm{HCl} \\
\text { d. h. in I Liter } \\
73 \mathrm{~g} \mathrm{HCl}\end{array}$ & $\begin{array}{c}18 \\
18 \\
95-100 \\
95-100\end{array}$ & $\begin{array}{r}\text { I } \\
\text { Io } \\
\text { I } \\
\text { 10 }\end{array}$ & $\begin{array}{l}0,1855 \\
0,0964 \\
0,1804 \\
0,1313\end{array}$ & $\begin{array}{l}0,1807 \\
0,0955 \\
0,1747 \\
0,1315\end{array}$ & $\begin{array}{l}97,4 \\
99,0 \\
96,8 \\
99,8\end{array}$ \\
\hline $\begin{array}{c}\text { o, i n. } H C l \\
\text { d. h. in I liter } \\
\text { 3,65 g HCl }\end{array}$ & $\begin{array}{c}18 \\
8 \\
95-100 \\
95-100\end{array}$ & $\begin{array}{r}1 \\
10 \\
1 \\
10\end{array}$ & $\begin{array}{l}0,1139 \\
0,0981 \\
0,0588 \\
0,0942\end{array}$ & $\mid \begin{array}{l}0,0138 \\
0,0944 \\
0,0586 \\
0,0927\end{array}$ & $\begin{array}{l}99,9 \\
96,2 \\
99,6 \\
98,4\end{array}$ \\
\hline $\begin{array}{l}2 \text { n. } \mathrm{H}_{2} \mathrm{SO}_{4} \\
\text { d. h. in I Liter } \\
98 \mathrm{~g} \mathrm{H}_{2} \mathrm{SO}_{4}\end{array}$ & $\begin{array}{c}18 \\
18 \\
95-100 \\
95-100\end{array}$ & $\begin{array}{r}\text { I } \\
\text { IO } \\
\text { I } \\
\text { IO }\end{array}$ & $\begin{array}{l}0, \text { IO33 } \\
0,11 \text { I I } 4 \\
0,0998 \\
0, \text { IOI } 4\end{array}$ & $\begin{array}{l}0,0994 \\
0,1056 \\
0,0927 \\
0,0940\end{array}$ & $\begin{array}{l}96,2 \\
94,7 \\
92,6 \\
92,7\end{array}$ \\
\hline $\begin{array}{l}\text { o, i n. } \mathrm{H}_{2} \mathrm{SO}_{4} \text {, } \\
\text { d. h. in I Liter } \\
4,9 \mathrm{~g} \mathrm{H}_{2} \mathrm{SO}_{4}\end{array}$ & $\begin{array}{c}I 8 \\
I 8 \\
95-100 \\
95-100\end{array}$ & $\begin{array}{r}1 \\
10 \\
1 \\
\text { 10 }\end{array}$ & $\begin{array}{l}0,0972 \\
0,1 \text { I I } 8 \\
0,0668 \\
0,1028\end{array}$ & $\begin{array}{l}0,0927 \\
0,1073 \\
0,0652 \\
0,0937\end{array}$ & $\begin{array}{l}95,3 \\
95,9 \\
97,6 \\
91,1\end{array}$ \\
\hline
\end{tabular}

$\mathrm{Zu}$ diesen Werten ist zu bemerken, dass die geringen Mengen vierwertigen Zinns keineswegs ihrem ganzen Betrage nach als vierwertig anodisch gelöst angenommen werden dürfen; denn einmal war die oxydierende Wirkung der Luft bei den Versuchen nicht ausgeschlossen und ferner konnte eine Oxydation durch entladene und nicht mit der Zinnanode in Reaktion getretene Anionen vor sich gehen. Bei verdünten Lösungen von Natriumsulfat oder Schwefelsäure und hohen Stromdichten beob- 
achtet man eine anodische Sauerstoffentwicklung, welche den entladenen und mit Wasser umgesetzten $\mathrm{SO}_{4}$-Anionen zuzuschreiben ist; dass nicht aller auf diesem Wege verfügbar gemachter Sauerstoff gasförmig entweicht, leuchtet ohne weiteres ein.

$Z$ inn geht also unter den verschiedensten Versuchsbedingungen in neutralen und sauren Elektrolyten wesentlich zweiwertig anodisch in Lösung; vierwertiges $Z$ inn bildet sich nur untergeordnet; die Menge des zweiwertigen beträgt stets über $90 \%$ der Gesamtmenge ${ }^{1}$ ).

II. Anodisches Verhalten des Antimons.

Die Versuche wurden ähnlich wie beim Zinn ausgeführt. Antimontrichlorid zerfrisst Baumwollgewebe, und als Hülle der Elektroden fand deshalb gehărtetes Filtrierpapier von der Firma Schleicher \& Schüll Verwendung, dessen Widerstandsfahigkeit allerdings auch nur sehr beschränkt war.

Elektrolyt: Chlornatriumlosung. Bei verdünnten Lösungen und Zimmerwärme bildet sich auf der Anode rasch eine graue Haut, wodurch der Strom fast ganz unterbrochen wird; die Grenze liegt bei einer $2 \mathrm{n}$. $\mathrm{NaCl}$-Lơsung. Diese gestattet zwar nicht bej Zimmerwärme, wohl aber bei etwa $100^{0}$ dem Strom den Durchgang und aberzieht sich mit einer lockeren Schicht von Antimonoxychlorid, frei von Verbindungen des fünfwertigen Antimons.

Bei einer 5 n. $\mathrm{NaCl}$-Lósung $(292,5 \mathrm{~g} \mathrm{NaCl}$ in I Liter), Temperaturen zwischen 18 und $100^{\circ}$ und Stromdichten zwischen 2 und Ioo Amp/qdm verwandelt sich ein Teil des Antimons in Antimonoxychlorid, weshalb der Elektrolyt stark alkalisch wird; ein anderer Teil bildet das ziemlich bestăndige Doppelsalz $3 \mathrm{NaCl} \cdot \mathrm{SbCl}_{3}$, das sich löst, um in der Năhe der Kathode unter Fallung von Hydroxyden zersetzt zu werden. Jedenfalls entstehen ausschliesslich Verbindungen des dreiwertigen Antimons.

Eine $6 \mathrm{n}$. $\mathrm{NaCl}$-Losung $\left(35^{\mathrm{I}} \mathrm{g} \mathrm{NaCl}\right.$ in I Liter) lieferte unter denselben wechselnden Versucbsbedingungen dieselben Ergebnisse wie eine $5 \mathrm{n}$. $\mathrm{NaCl}$-Lösung mit dem einzigen Unterschied, dass der Elektrolyt nur sehr schwach alkalische Reaktion annahm, weil die hydrolytische Spaltung des $\mathrm{SbCl}_{3}$ sebr gering blieb.

1) Mit diesem Befunde steht eine Angabe von R. Lorenz (Zeitschr. f. anorgan. Chemie 12,436) in Widerspruch, wonach sich mit $\mathrm{KCl}$ als Elektrolyten leicht Orthozinnsäure gewinnen lässt; diese Angabe ist auch in die „Uebungsbeispiele für elektrolytische Darstellung chemischer Präparate" vou K. E1bs übergegangen (S. 42). Vermutlich liegt dieser unrichtigen Angabe nicht eine falsche Beobachtung, sondern ein Schreibfehler oder ein Versehen zu Grunde, was bei der Zweideutigkeit des Wortes „Zinnoxydhydrat" leicht begreiflich erscheint.
Der Vorgang bei der Elektrolyse bestand in der fast quantitativen Bildung von $\mathrm{SbCl}_{3}$ an der Anode, welches als $3 \mathrm{NaCl} \cdot \mathrm{SbCl}_{3}$ in Lósung geht und an der Kathode durch die dort in äquivalenter Menge zur Verfügung stehende Natronlauge ausgefallt wird. Die zuerst entstehende orthoantimonige Säure $S b(O H)_{3}$ ist unbeständig und geht in metantimonige Säure $\mathrm{SbO} \cdot \mathrm{OH}$ uber; aber auch diese gibt um so mehr, je boher die Temperatur ist, Wasser ab unter Bildung von Antimontrioxyd. So enthielt das bei ${ }_{1} 8^{0}$ aus der $6 \mathrm{n}$. $\mathrm{NaCl}$-Lösung ausgefallene Hydroxyd $79,5 \% S b$, das bei $60^{0}$ gefallene $80,7 \% S b$, das bei $80^{\circ}$ ausgeschiedene $81,5 \% S b$, wăhrend der $S b$-Gehalt der metantimonigen Säure $\mathrm{OSbOH} 78,4 \%$, der des Antimontrioxyds $\mathrm{Sb}_{2} \mathrm{O}_{3} \quad 83,3 \%$ beträgt.

Elektrolyt: Natriumsulfatlösung. Wie vorauszusehen war, trat bei $1 / 10 \mathrm{n}$. und $2 \mathrm{n}$. $\mathrm{Na}_{2} \mathrm{SO}_{4}$-Lösungen sofort Stromunterbrechung ein, weil sich ein äusserst dünner, nichtleitender Ueberzug von basischem $S b$-Sulfat auf der Anode bildete.

Auf Grund dieses Verhaltens kann man ein sogen. Stromventil bauen, eine Vorrichtung, die den Strom nur in einer bestimmten Richtung durchlässt. Ein Glasgefäss enthält in einer I n. Natriumsulfatlosung als Elektroden eine $P b$-Platte und einen $S b$-Stab. Fliesst der positive Strom in der Richtung vom Blei zum Antimon, so geht er ungehindert durch den Apparat; schaltet man den Strom in umgekehrter Richtung ein, so wird er augenblicklich unterbrochen, fliesst aber beim .Umschalten wieder ungestört. Die Wirksamkeit der Vorrichtung wurde nur bis zu Spannungen von I 8 Volt erprobt.

Elektrolyt: Salzsäure. Die Lösung der $S b$-Anode erfolgt in genügend konzentrierter Salzsăure ohne Störung; das Antimon löst sich zu $\mathrm{SbCl}_{3}$, welches in Schlieren heruntersinkt. Bei stark wechselnden Stromdichten, Temperaturen und Săurekonzentrationen wurde stets Trichlorid, niemals Pentachlorid erhalten. Bei $1 / 10 \mathrm{n}$. bis zu $3 \mathrm{n} . \mathrm{HCl}$ trat mehr oder minder erhebliche Hydrolyse des $\mathrm{SbCl}_{3}$ ein; erst bei $4 \mathrm{n} . \mathrm{HCl}$ verlief die Losung der Anode glatt.

Elektrolyt: Schwefelsăure. Alle unter den verschiedensten Bedingungen angestellten Versuche lieferten immer nur ein Ergebnis, nämlich rasche Stromunterbrechung infolge der Bildung einer dünnen, grauen, nichtleitenden Haut auf der Anode. Ob diese Haut bei verdannter $\mathrm{H}_{2} \mathrm{SO}_{4}$ aus basischen Sulfaten, bei konzentrierterer aus darin unlóslichem Sulfat bestand, konnte nicht festgestellt werden, weil der Ueberzug sich nicht in fassbarer Menge bildete. 


\section{Anodisches Verhalten des Wismuts.}

Elektrolyt: Chlornatriumlossung. In verdünnten Lösungen tritt hydrolytische Spaltung des entstandenen $\mathrm{BiCl}_{3}$ ein, erst bei $6 \mathrm{n} . \mathrm{NaCl}$ Lösung erfolgt klare Auflossung der Anode bei allen Temperaturen zwischen 20 und $100^{\circ}$. Die durch die kathodische Natronlauge ausgefallten Hydrate waren niemals chlorfrei. Unter keinen Umständen konnte die Bildung einer Verbindung des fünfwertigen Wismuts beobachtet werden. Die hydrolytischen Spaltungsprodukte setzten sich stets locker auf der Anode ab, so dass die Stromleitung ziemlich ungestört blieb.

Elektrolyt: Natriumsulfatlösung. Bei verschiedenen Stromdichten, Temperaturen und Konzentrationen wurde der Strom sofort durch Bildung einer grauen, nicht leitenden Haut auf der Anode unterbrochen. Da Wismutsulfat mit Kalium- und Ammoniumsulfat, nicht aber mit Natriumsulfat lösliche Doppelsalze bildet, so lag die Moglichkeit vor, dass in den Sulfaten von $\mathrm{K}$ und $\mathrm{NH}_{4}$ die Wismutanode in Lösung ging; tatsächlich ist dies jedoch nicht der Fall.

Elektrolyt: Salzsäure. In stark verdünnter Säure bedeckt sich die Anode mit Oxychlorid, welches jedoch die Stromleitung nicht verhindert; in $3 \mathrm{n} . \mathrm{HCl}$ und noch stärkerer Salzsäure tritt glatte Lösung ein, und die Anode bleibt blank. Verbindungen des fünfwertigen Wismuts liessen sich nie nachweisen.

Elektrolyt: Schwefelsäure. Unter den verschiedensten Versuchsbedingungen bildet sich stets eine dünne graue Haut auf der Anode, welche nach kurzer Zeit Unterbrechung des Stromes veranlasst.

\section{Zusammenfassung.}

I. Unter Versuchsbedingungen, die bezüglich Stromdichte, Temperatur und Konzentration in sehr weiten Grenzen schwanken, geht in den Elektrolyten Natriumchlorid, Natriumsulfat, Salzund Schwefelsäure das $Z$ inn stets zum weitaus grössten Teil zweiwertig anodisch in Lossung.

2. Von den obigen Elektrolyten eignen sich für Antimon als Lösungsanode nur Natriumchlorid und Salzsäure als Elektrolyte. Es entstehen ausschliesslich Verbindungen des dreiwertigen Antimons.

3. Das Wismut verhält sich als Anode dem Antimon ăhnlich; es bilden sich nur Verbindungen des dreiwertigen Wismuts.

Giessen, im April r 904.

Physikalisch-Chemisches Univers-Laboratorium. (Eingegangen: 22. April.)

\section{BEMERKUNG ÜBER IONENREAKTIONEN IN DER ORGANISCHEN CHEMIE.}

\section{Von Walther Löb.}

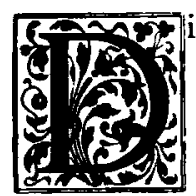
in dieser Zeitschrift kürzlich erschienene Arbeit von Franz Goldschmidt ${ }^{1}$ : "Zur Theorie der Verseifung", veranlasst mich, auf einen früheren Vortrag ${ }^{2}$ ) von mir hinzuweisen, der wegen seines allgemein gebaltenen Titels schon mebrfach bei Behandlung des in der Ueberschrift genannten Themas übersehen worden ist. So hat auch Walden ${ }^{3}$ ), welcher die in der Literatur angegebenen Aeusserungen über die Bedeutung der Ionenlehre für organische Reaktionen möglichst vollständig wiedergibt, jene Arbeit von mir nicht citiert, ebenso wenig wie Abegg und Bodländer ${ }^{4}$ ) und Euler ${ }^{5}$.

In meinem Vortrage crörterte ich speziell die Annahme, dass auch die organischen Reak-

1) Zeitschr. f. Elektrochemie 10, 22: (1904).

2) Die Bedeutung der Elektrochemie für die organische Chemie. Zeitschr. f. Elektrochemie 3, 42 (I896).

3) Zeitschr. f. physik. Chemie 43, 385 (I903).

4) Zeitschr. f. anorg. Chemie 20, 495 (1899).

5) Ber. d. Deutsch. chem. Ges. 33, 3202 (1900); Zeitschr. f. physik. Chemie 36, 405 (I9OI). tionen Ionenreaktionen sind. Diese mir damals notwendig erscheinende Folgerung der Dissociationstheorie führte ich für einige experimentell behandelte Falle näher aus, und zwar für die Esterbildung und die Reduktion der Nitrokörper.

Zur Deutung der Esterbildung im Lichte der Dissociationstheorie wurde, wie dies Euler, Walden und Goldschmidt tun, eine zweifache Dissociation der Säure angenommen, und zwar nebeneinander eine Wasserstoff - und eine Hydroxyldissociation, so dass eine Säure als ein amphoterer Elektrolyt erscheint $\left.{ }^{1}\right)$ :

$\mathrm{RCOOH} \rightarrow \mathrm{RCOO}+\mathrm{H}^{\circ}$ und $\mathrm{RCO}^{\circ}+\mathrm{OH}^{\circ}$.

Das Anion $R C O O^{\prime}$ repräsentiert mit dem Kation $R C O^{\circ}$ das Säureanhydrid, das mit dem wie eine schwache Säure wirkenden Alkohol zum Ester zusammentritt:

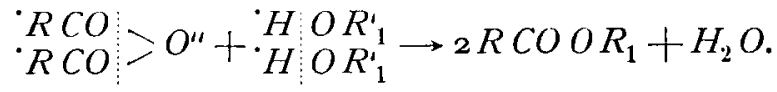

I) Siehe auch Walker, Proc. Roy. Soc. 73, I55 (Igo,); Zeitschr. f. Elektrochemie 10, 187 (1904), Referat von A begg. 